\title{
Mitochondrial Complex I Inhibition by Metformin Limits Reperfusion Injuryฐ
}

\author{
Ahmed A. Mohsin, ${ }^{1}$ Qun Chen, ${ }^{1}$ Nanhu Quan, Thomas Rousselle, Michael W. Maceyka, \\ Arun Samidurai, Jeremy Thompson, Ying $\mathrm{Hu}$, Ji Li, and Edward J. Lesnefsky \\ Department of Biochemistry and Molecular Biology (A.A.M., M.W.M., E.J.L.) and Pauley Heart Center, Division of Cardiology, \\ Department of Internal Medicine (Q.C., A.S., J.T., Y.H., E.J.L.), Virginia Commonwealth University, Richmond, Virginia; Department \\ of Physiology and Biophysics, University of Mississippi Medical Center, Jackson, Mississippi (N.Q., T.R., J.L.); and Cardiology \\ Section Medical Service, McGuire Veterans Affairs Medical Center, Richmond, Virginia (E.J.L.)
}

Received December 14, 2018; accepted February 26, 2019

\begin{abstract}
Transient, reversible blockade of complex I during early reperfusion after ischemia limits cardiac injury. We studied the cardioprotection of high dose of metformin in cultured cells and mouse hearts via the novel mechanism of acute downregulation of complex I. The effect of high dose of metformin on complex I activity was studied in isolated heart mitochondria and cultured $\mathrm{H} 9 \mathrm{c} 2$ cells. Protection with metformin was evaluated in $\mathrm{H} 9 \mathrm{c} 2$ cells at reoxygenation and at early reperfusion in isolated perfused mouse hearts and in vivo regional ischemia reperfusion. Acute, high-dose metformin treatment inhibited complex I in ischemia-damaged mitochondria and in H9c2 cells following hypoxia. Accompanying the complex I modulation, high-dose metformin at reoxygenation decreased death in $\mathrm{H} 9 \mathrm{c} 2$
\end{abstract}

cells. Acute treatment with high-dose metformin at the end of ischemia reduced infarct size following ischemia reperfusion in vitro and in vivo, including in the AMP kinase-dead mouse. Metformin treatment during early reperfusion improved mitochondrial calcium retention capacity, indicating decreased permeability transition pore (MPTP) opening. Acute, high-dose metformin therapy decreased cardiac injury through inhibition of complex I accompanied by attenuation of MPTP opening. Moreover, in contrast to chronic metformin treatment, protection by acute, high-dose metformin is independent of AMPactivated protein kinase activation. Thus, a single, high-dose metformin treatment at reperfusion reduces cardiac injury via modulation of complex I.

\section{Introduction}

Acute coronary syndromes caused by the abrupt occlusion of a coronary artery leading to ST segment elevation myocardial infarction (STEMI) remain a major clinical challenge. Despite timely and successful reperfusion, substantial myocardial

This work was supported by the Office of Research and Development, Medical Research Service Merit Review Award (2IO1BX001355-01A2), Department of Veterans Affairs (E.J.L.); National Institutes of Health [Grants R21AG054975-01 (Q.C.), R01GM124108 (J.L.), and R01AG049835 (J.L.)]; American Diabetes Association 1-17-IBS-296 (J.L.); and Pauley Heart Center, Virginia Commonwealth University (Q.C., E.J.L.)

A portion of this work was previously presented as meeting abstracts in the following: Mohsin A, Chen Q, Quan N, Maceyka M, Samidurai A, Thompson J, $\mathrm{Hu}$ Y, Li J, and Lesnefsky EJ (2016) Cardioprotection during early reperfusion via complex I inhibition by metformin. Circulation Abstract M1123.

Mohsin AA, Maceyka M, and Lesnefsky EJ (2018) Modulation of electron transport by metformin in cardiac protection: role of complex I. Society for Heart and Vascular Metabolism Sixteenth Annual Scientific Sessions; 2018 October; Charleston, SC.

Thesis Information: A portion of this work was included in the Doctoral Thesis of the Department of Biochemistry, Virginia Commonwealth University, of Dr. Ahmed A. Mohsin, entitled "Modulation of electron transport by metformin in cardiac protection: role of complex I."

${ }^{1}$ A.A.M. and Q.C. contributed equally to this work.

https://doi.org/10.1124/jpet.118.254300.

S This article has supplemental material available at jpet.aspetjournals.org. injury still occurs. A significant portion of the cardiac injury occurs during the early reperfusion phase, after coronary flow has been restored (Yellon and Hausenloy, 2007). The extent of injury from even successfully reperfused STEMI places patients at risk for transition to ischemic cardiomyopathy and heart failure.

Damage to cardiac mitochondria is a key mechanism of cardiac injury during ischemia and reperfusion (Gustafsson and Gottlieb, 2008; Lesnefsky et al., 2017). Ischemia, especially periods associated with STEMI, result in damage to the mitochondrial electron transport chain (ETC) (Lesnefsky et al., 2017). The ischemia-damaged ETC enhances cardiac injury during early reperfusion (Lesnefsky et al., 2004b). ETC-dependent processes that injure the myocardium include the increased generation of reactive oxygen species (ROS) (Kevin et al., 2003), opening of the mitochondrial permeability transition pore (MPTP) (Gomez et al., 2007), and release of mitochondrial proteins that activate cell death (Weiss et al., 2003). Surprisingly, the bulk of damage to the ETC occurs during ischemia and persists into reperfusion (Lesnefsky et al., 2004b). Blockade of electron transport immediately before ischemia robustly limits ischemia-induced mitochondrial damage and allows reperfusion to occur with essentially normal mitochondria

ABBREVIATIONS: AMPK, AMP-activated protein kinase; AMPK KD, AMPK kinase-dead; CRC, calcium retention capacity; DMEM, Dulbecco's modified Eagle's medium; ETC, electron transport chain; LAD, left anterior descending; MOPS, 3-(N-morpholino)propanesulfonic acid; MPTP, mitochondrial permeability transition pore; RO, reoxygenation; ROS, reactive oxygen species; SI, simulated ischemia; STEMI, ST elevation myocardial infarction; TTC, 2,3,5-triphenyltetrazolium chloride. 
leading to substantially reduced infarct size (Lesnefsky et al., 2017). Thus, reperfusion with ischemia-damaged mitochondrial increases cardiac injury (Murphy, 2004; Lesnefsky et al., 2017). Because treatment before ischemia is not feasible in clinical settings, clinically relevant adjunctive pharmacologic treatment to limit cardiac injury is focused on the modulation of metabolism in the ischemia-damaged mitochondria during early reperfusion.

The modulation of complex I activity using physiologic or pharmacological approaches at the onset of reperfusion decreases cardiac injury (Stewart et al., 2009; Chouchani et al., 2013; Xu et al., 2014). The protective mitochondrial phenotype consists of a partial blockade of complex I that preserves mitochondrial membrane potential (Szczepanek et al., 2011). Thus, translational utility of complex I inhibition should focus on reversible, modest blockade rather than a robust inhibition. Following ischemia, decreased complex I activity occurs due both to direct structural damage (Chen et al., 2008) and to a reversible ischemia-induced conformational change to a deactive form (Galkin et al., 2009). Complex I exists in two forms: A form (active form) and D form (deactive form). During ischemia, complex I transitions to the D form (Galkin et al., 2009; Gorenkova et al., 2013). Ischemia-altered complex I contributes to injury during early reperfusion (Lesnefsky et al., 2017). Thus, an agent with selectivity toward ischemia-altered complex I would provide optimal pharmacologic intervention during early reperfusion, potentially blunting complex I-mediated injury during reperfusion while minimizing inhibition of complex I in myocardium that has not sustained damage.

Metformin belongs to the biguanide family (Mamputu et al., 2003) and is an activator of the AMP kinase (AMPK) signaling pathway (Calvert et al., 2008). Chronic metformin activates AMPK through a mild complex I inhibition. Complex I is more sensitive to biguanide-mediated inhibition when in the $\mathrm{D}$ form (Matsuzaki and Humphries, 2015). We proposed that the acute administration of high-dose metformin during early reperfusion can achieve significant complex I inhibition, especially in ischemia-altered mitochondria. The goal of the current study is to use transient, high-dose metformin treatment to reversibly block complex I immediately following ischemia and to test the hypothesis that inhibition of ischemia-altered complex I during reperfusion will attenuate the rapid reactivation of complex I that is associated with reperfusion injury to the myocardium. We posit that, although metformin is a known AMPK activator, acute, high-dose treatment will lead to acute cardioprotection via complex I inhibition independent of activation of AMPK.

\section{Materials and Methods}

Reagents. The reagents used in isolation of mitochondria and mitochondria-related functional assays were purchased from SigmaAldrich (St. Louis, MO). Antibodies (AMPK $\alpha$ 2532S, P-AMPK $\alpha$ 2531S, acetyl-CoA carboxylase 3661, and phosphorylated-acetyl-CoA carboxylase 3662) were purchased from Cell Signaling Technology (Danvers, MA). Mitochondrial CytoTox-Fluor Cytotoxicity Assay G8000 was obtained from Promega (Madison, WI).

Cell Culture and Simulated Ischemia Reoxygenation. H9c2(2-1) (American Type Culture Collection CRL-1446) mycoplasma-free cardiac myoblasts were grown in complete Dulbecco's modified Eagle's medium (DMEM; Sigma-Aldrich) containing $4.5 \mathrm{mM}$ glucose and supplemented with $10 \%$ fetal bovine serum and $1 \%$ penicillin and streptomycin.
Briefly, cells $\left(3 \times 10^{4}\right.$ cells $\left./ \mathrm{ml}\right)$ were seeded in $25-\mathrm{cm}^{2}$ flasks after reaching $70 \%$ confluency. Cells were exposed to simulated ischemia (SI) by replacing the culture medium with ischemia buffer (Das et al., 2005) and incubating in the Galaxy $\mathrm{O}_{2}$ controlled incubator at $5 \% \mathrm{CO}_{2}$ and $1 \% \mathrm{O}_{2}$ at $37^{\circ} \mathrm{C}$ for 6 hours. Reoxygenation (RO) was performed by replacing ischemia buffer with complete DMEM under aerobic cell culture condition $\left(5 \% \mathrm{CO}_{2}\right.$ and $\left.19 \% \mathrm{O}_{2}\right)$ at $37^{\circ} \mathrm{C}$ with or without treatment with metformin. Metformin treatment $(1 \mathrm{mM})$ was used at the onset of RO, continued for 30 minutes, and then replaced with media containing DMEM without metformin. RO was continued for 24 hours. Other experiments used metformin treatment of the entire RO period. Assessment of cell death was performed by measurement of dead cell protease activity, according to manufacturer instruction, by using the CytoTox-Fluor Cytotoxicity Assay (Promega). This assay uses a fluorogenic peptide substrate (bis-alanyl-alanyl-phenylalanylrhodamine 110) that labels cells that have lost membrane integrity (Jochmans et al., 2012). Using a 96-well plate seeded with 5000 cells/well, separate plates were used in parallel: one plate for cells incubated under normoxia condition as a control in parallel with additional plates of cells that underwent 6-hour SI, followed by 24-hour RO. At the end of the experiment, assay reagent $(5 \times)$ was added to all wells (20 $\mu \mathrm{l}$ per well), followed by brief mixing using orbital shaking, and then incubated for 30 minutes at $37^{\circ} \mathrm{C}$, followed by measurement of resulting fluorescence (485 nm Ex/520 nm Em).

High-Resolution Respirometry-Oroboros. Measurements were made in MiR05 medium at $37^{\circ} \mathrm{C}$. H9c2 cells treated or control were added into MiR05 medium, pH 7.1 (Strub et al., 2011). Briefly, cells were harvested following the incubation period and resuspended in $2.1 \mathrm{ml}$ MiR-05 pH.7.1. Cell density was determined by counting the cells using a hemocytometer. For measurement of endogenous respiration, glutamatepyruvate-malate $(10,5$, and $2 \mathrm{mM}$, respectively) were used as the substrate of complex I. Permeabilization of H9c2 cells by digitonin $(10 \mu \mathrm{g} / \mathrm{ml})$ was followed by addition of $1 \mathrm{mM}$ ADP. Next, inhibition of complex I by rotenone $(0.05 \mu \mathrm{M})$ was followed by addition of succinate $(10 \mathrm{mM})$ as substrate for respiration of complex II. Thenoyltrifluoroacetone $(40 \mu \mathrm{M})$ was then added to inhibit complex II respiration; complex IV respiration was measured by addition of $N, N, N^{\prime}, N^{\prime}$-tetramethyl$p$-phenylenediamine/ascorbate $(0.3 / 3 \mathrm{mM})$. Finally, azide $(15 \mathrm{mM})$ was added as a complex IV inhibitor.

Hypoxia RO experiments were performed in the Oroboros using a similar approach, except that after the addition of ADP, the cells were allowed to consume all the oxygen in the chamber. Ten minutes of hypoxia was started at the time of zero oxygen concentration with no evident oxygen consumption. RO was performed by opening the chamber, and RO documented by measurement of the oxygen content. Respiration rate was monitored for several minutes, the rate determined, and rotenone added to derive the background rate for complex I. Samples were studied with or without $1 \mathrm{mM}$ metformin added after the initial addition of ADP.

Preparation of Mouse Hearts for Perfusion. The Animal Care and Use Committees of the McGuire VA Medical Center and Virginia Commonwealth University approved the study. Studies were carried out in accordance with the Guide for the Care and Use of Laboratory Animals as adopted and promulgated by the National Institutes of Health. Hearts from male C57BL/6 mice (2 to 3 months of age) were excised and perfused retrograde via the aorta in the Langendorf mode with modified Krebs-Henseleit buffer ( $115 \mathrm{mM} \mathrm{NaCl}, 4.0 \mathrm{mM}$ $\mathrm{KCl}, 2.0 \mathrm{mM} \mathrm{CaCl}_{2}, 26 \mathrm{mM} \mathrm{NaHCO}_{3}, 1.1 \mathrm{mM} \mathrm{MgSO}_{4}, 0.9 \mathrm{mM} \mathrm{KH}_{2} \mathrm{PO}_{4}$, and $5.5 \mathrm{mM}$ glucose), gassed with $95 \% \mathrm{O}_{2}-5 \% \mathrm{CO}_{2}$, as previously described (Chen et al., 2011). Hearts were paced via epicardial leads at 420 beats per minute. Pacing was suspended during ischemia and during the initial 20 minutes of reperfusion. Cardiac function was monitored with a balloon inserted into the left ventricle (Powerlab; AD Instruments, Colorado Springs, CO). Time control hearts were perfused without ischemia. Hearts were perfused for 15-minute equilibration with Krebs-Henseleit buffer, followed by 25-minute global ischemia at $37^{\circ} \mathrm{C}$ and 30 - or 60 -minute reperfusion. In the metformin treatment group, metformin ( $2 \mathrm{mM}$ final concentration) was perfused for 
5 minutes at the onset of reperfusion, followed by normal buffer perfusion. Cardiac injury was assessed by the measurement of infarct size at 60-minute reperfusion with 2,3,5-triphenyltetrazolium chloride (TTC) and the release of lactate dehydrogenase into coronary effluent during the entire reperfusion period (Das et al., 2005). Cardiac mitochondria were isolated after 30 minutes of reperfusion in separate experiments.

Isolation and Study of Mouse Heart Mitochondria. Cardiac mitochondria were isolated, as previously described (Chen et al., 2011). Mouse hearts were placed in cold buffer A [composition in millimolar: $100 \mathrm{KCl}, 50$ 3-(N-morpholino)propanesulfonic acid (MOPS), 1 EGTA, $5 \mathrm{MgSO}_{4}$, and $1 \mathrm{ATP}$, blotted dry, weighed, and homogenized using a polytron tissue homogenizer at $10,000 \mathrm{rpm}$ for 2.5 seconds in the presence of trypsin ( $5 \mathrm{mg} / \mathrm{g}$ tissue). The homogenate was incubated for 15 minutes at $4^{\circ} \mathrm{C}$; then the same volume of buffer $\mathrm{B}$ (buffer $\mathrm{A}+0.2 \%$ bovine serum albumin) was added, the mixture was centrifuged at $500 \mathrm{~g}$ for 10 minutes to pellet myofibrillar debris, and the supernatant again was centrifuged at $3000 \mathrm{~g}$ to pellet mitochondria. The mitochondrial pellet was first washed with buffer B, resuspended in KME (100 mM $\mathrm{KCl}, 50 \mathrm{mM}$ MOPS, $0.5 \mathrm{mM}$ EGTA), centrifuged at $3000 \mathrm{~g}$ to yield the final mitochondrial pellet, and resuspended in the same buffer for study (Chen et al., 2011). Protein content was measured using the Lowry method. Mitochondria were kept on ice and used within 4 hours.

The rate of oxygen consumption in mitochondria was measured using a Clark-type oxygen electrode at $30^{\circ} \mathrm{C}$, as previously described (Chen et al., 2011). Mitochondria were incubated in $80 \mathrm{mM} \mathrm{KCl}$, $50 \mathrm{mM}$ MOPS, $1 \mathrm{mM}$ EGTA, $5 \mathrm{mM} \mathrm{KH_{2 }} \mathrm{PO}_{4}$, and $1 \mathrm{mg}$ defatted, dialyzed bovine serum albumin/ml at $\mathrm{pH}$ 7.4. Glutamate $(20 \mathrm{mM})+$ malate $(10 \mathrm{mM})$ (complex I substrate) and succinate $(20 \mathrm{mM})$ plus $7.5 \mu \mathrm{M}$ rotenone (complex II substrate) were studied. The activities of complex I, II, and citrate synthase were measured in detergentsolubilized mitochondria using previously described methods (Chen et al., 2011). To test the effect of metformin on the activities of complex I and II, metformin was present in the assay.

Calcium Retention Capacity in Isolated Mitochondria. Calcium retention capacity (CRC) was used to assess calciuminduced MPTP opening in isolated mitochondria, as previously described (Paillard et al., 2009). CRC was evaluated in mitochondria $(0.25 \mathrm{mg})$ incubated in medium containing $150 \mathrm{mM}$ sucrose, $50 \mathrm{mM} \mathrm{KCl}, 2 \mathrm{mM} \mathrm{KH}_{2} \mathrm{PO}_{4}$, and $5 \mathrm{mM}$ succinic acid in $20 \mathrm{mM}$ Tris/HCl, $\mathrm{pH}$ 7.4, by sequential pulses of $5 \mathrm{nmol}$ calcium. Extramitochondrial $\mathrm{Ca}^{2+}$ concentration was estimated using $0.5 \mu \mathrm{M}$ Calcium Green-5N (Life Technology, Eugene, OR) and fluorescence monitored with excitation and emission wavelengths set at 500 and $530 \mathrm{~nm}$, respectively.

Western Blotting. H9c2 cells were trypsinized and then resuspended in complete DMEM and centrifuged, and cell pellets were washed twice using phosphate-buffered saline. Pellets were resuspended in $1 \times$ lysis buffer (Cell Signaling) supplemented with protease and phosphatase inhibitor cocktails (Roche, Indianapolis, IN) and incubated on ice for 10 minutes, after which samples were centrifuged at $12,000 \mathrm{~g}$ for 10 minutes at $4^{\circ} \mathrm{C}$ to remove insoluble cell debris. The supernatant was collected, and protein was measured using the Lowry method. Cell samples or cytosol samples from mouse hearts were combined with $2 \times$ Laemmli sample buffer (161-0737; Bio-Rad, Hercules, CA) and boiled for 5 minutes, after which proteins were separated using SDS-PAGE on 4-20\% tris-glycine extended gradient gels (Bio-Rad) and transferred to Immobilion-P transfer membrane paper (Millipore, Burlington, MA). After blocking nonspecific binding sites, membranes were incubated with the primary antibodies at $4^{\circ} \mathrm{C}$ overnight. The next day, the blots were washed three times, 10 minutes each, with Tris-buffered saline $(25 \mathrm{mM}$ Tris, $150 \mathrm{mM}$ $\mathrm{NaCl}, 2 \mathrm{mM} \mathrm{KCl}$ ), pH 7.4 7.6, including $0.1 \%$ Tween as detergent, and were incubated with a 1:10,000 dilution of anti-mouse or antirabbit IgG $\mathrm{F}(\mathrm{ab})_{2}$ fragments conjugated with horseradish peroxidase in $2 \%$ bovine serum albumin in Tween-buffered saline for 1 hour. Lastly, the blots were washed three times with Tris-buffered saline with $0.1 \%$ Tween and developed using Amersham ECL Plus Western blotting detection reagents (GE Healthcare, UK) and imaged by ChemiDoc gel imaging system (Bio-Rad). Protein bands were quantified by densitometry analysis by using ImageJ software (National Institutes of Health, Bethesda, MD).

In Vivo Murine Model of Ischemia and Reperfusion with and without Metformin Treatment. The Animal Care and Use Committee of the University of Mississippi Medical Center approved the study. Studies were carried out in accordance with the Guide for the Care and Use of Laboratory Animals as adopted and promulgated by the National Institutes of Health. In vivo regional ischemia and reperfusion were performed, as previously described (Quan et al., 2017). Wild-type mice (C57BL/6) were anesthetized with isoflurane (2\%) and placed on a ventilator (Harvard Apparatus, Holliston, MA). Core temperature was maintained at $37^{\circ} \mathrm{C}$ with a heating pad. After left lateral thoracotomy, the left anterior descending (LAD) coronary artery was occluded for 45 minutes and then reperfused for 24 hours. An electrocardiogram and blanching of left ventricle confirmed ischemia in the risk area with ST-segment elevation on electrocardiogram during coronary occlusion ( $\mathrm{AD}$ Instruments). Before the end of ischemia, metformin was administered i.v. to achieve $2 \mathrm{mM}$ final concentration in blood volume in the metformin-treated group at the onset of reperfusion. The mouse was either subjected to $75 \mu \mathrm{l} 40 \mathrm{mM}$ metformin i.v. infusion or a matched-volume saline control. A $75 \mu \mathrm{l} 40 \mathrm{mM}$ metformin i.v. will equilibrate to $2 \mathrm{mM}$ total body concentration under the assumption of $1.5 \mathrm{ml} \mathrm{blood} / \mathrm{mouse}$. After 24 hours, the mice were anesthetized and hearts excised and stained with TTC and Evans blue dye to delineate the extent of myocardial necrosis as a percentage of the ischemic area at risk. The heart and aorta dissected free, mounted onto a $20 \mathrm{~g}$ cannula, the heart is perfused with $37^{\circ} \mathrm{C} 1 \%$ TTC and incubated in $1 \%$ TTC solution. Postincubation, the LAD was religated, and the noninfarcted area was then stained with $1 \%$ Evans Blue via the aortic cannula. Hearts were then fixed in $10 \%$ phosphate-buffered formalin for 12-16 hours, sectioned axially into 1-mm-thick slices, photographed with a Leica microscope (Leica Microsystems, Wetzlar, Germany), and analyzed with ImageJ software (National Institutes of Health) (Quan et al., 2017). Wild-type littermates or AMPK kinasedead (AMPK KD) mice on a C57BL/6 background (Russell et al., 2004; Yang et al., 2016) underwent regional ischemia and reperfusion in a similar manner (Ma et al., 2010). The AMPK KD mice express a kinase-dead subunit $\alpha 2 \mathrm{~K} 45 \mathrm{R}$ mutation, driven in heart and skeletal muscles by the muscle creatine kinase promoter on a C57BL/6J background (Russell et al., 2004; Yang et al., 2016). The mice used for study were from a colony in Dr. Ji Li's laboratory.

Statistical Analysis. Data are expressed as the mean \pm S.E.M. (Steel and Torrie, 1960). For all analyses, differences between groups ( $\geq 3$ groups) were compared by one-way analysis of variance (SigmaStat 3.5, Systat, Richmond, CA; and GraphPad Prism version 7.00 for Windows, GraphPad Software, La Jolla, CA). When a significant $F$ value was obtained, means were compared using the StudentNewman-Keuls test of multiple comparisons. Differences between two groups were compared by unpaired Student $t$ test (SigmaStat 3.5; Systat). Statistical significance was defined as a value of $P<0.05$.

\section{Results}

Partial Inhibition of Complex I Respiration by High Dose of Metformin Treatment. Complex I activity was measured in mitochondria isolated from control or ischemic mouse hearts in the presence or absence of the metformin. Compared with control, 25-minute global ischemia decreased complex I activity in mouse heart mitochondria [mean \pm S.E.M., control (795 $\pm 65 \mathrm{mU} / \mathrm{min}$ per milligram mitochondrial protein, $n=3$ ) vs. ischemia (582 $\pm 44, n=3), P<0.05$ ] (Fig. 1A). Ischemia did not alter the activity of complex II (Fig. 1B). Complex I activity was not altered in nonischemic control mitochondria in the presence of acutely administered metformin $(2 \mathrm{mM})$ [mean \pm S.E.M., control $(795 \pm 65 \mathrm{mU} / \mathrm{min}$ per milligram mitochondrial protein, $n=3$ ) vs. control with $2 \mathrm{mM}$ metformin 

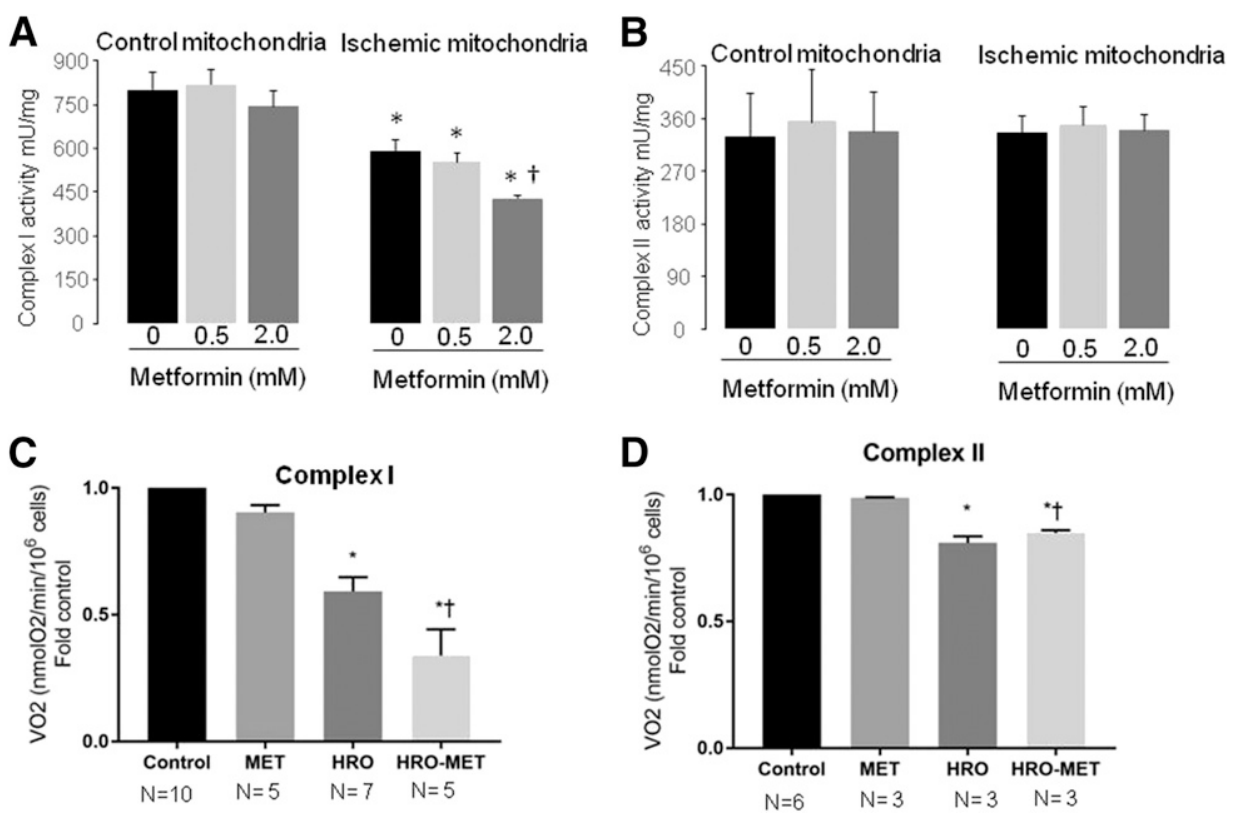

Fig. 1. Metformin inhibited complex I activity in heart mitochondria following ischemia. (A) Compared with nonischemic time control, ischemia decreased complex I activity in mitochondria isolated from mouse hearts at the end of 25 minutes of stop flow ischemia. Acute exposure to metformin of 0.5-2.0 mM resulted in an additional decrease in complex I activity that was selective to mitochondria isolated from hearts at the end of ischemia, whereas complex I activity in mitochondria from nonischemic control hearts was not downregulated. (B) In contrast to complex I, the activity of complex II was not affected by acute, high-dose exposure to metformin. (C) In parallel to isolated mitochondria, acute, high-dose exposure to metformin (1 mM) decreased the rate of oxidative phosphorylation with complex I substrates in H9c2 cells during RO after hypoxia (HRO-MET) compared with hypoxia RO alone (HRO). In contrast, acute treatment with $1 \mathrm{mM}$ metformin did not affect complex I-mediated respiration in cells not exposed to HRO. (D) In contrast to complex I-mediated respiration, acute metformin exposure did not downregulate the rate of oxidative phosphorylation with complex II substrates, again indicating the selectivity of metformin-induced downregulation of respiration to complex I. All data are expressed as mean \pm S.E.M.; $* P<0.05$ vs. nonischemic control; ${ }^{\dagger} P<0.05$ vs. nonmetformin treated; $n=3$ in each group for mitochondria (A and $\left.\mathrm{B}\right)$ and number of individual experiments shown for cell studies.

$(740 \pm 54, n=3), p=$ N.S.]. However, complex I activity was further decreased in ischemia-altered mitochondria in the presence of $2 \mathrm{mM}$ metformin [mean \pm S.E.M., ischemia $(582 \pm 44 \mathrm{mU} / \mathrm{min}$ per milligram mitochondrial protein, $n=3$ ) vs. ischemia $+2 \mathrm{mM}$ metformin $(424 \pm 12, n=3), P<0.05$ ] (Fig. 1A). Metformin did not decrease complex II activity in either control or postischemic mitochondria (Fig. 1B).

Metformin inhibited respiration with complex I substrates in H9c2 cells following 10-minute hypoxia (Fig. 1C). Hypoxia RO led to a decrease in oxidative phosphorylation in H9c2 cells (Fig. 1D). Metformin did not lead to additional inhibition of respiration with complex II (Fig. 1D) or complex IV (data not shown) substrates. These findings indicate that acute, highdose metformin treatment selectively inhibits electron transport at complex I. Moreover, inhibition is acutely evident in posthypoxia cells and postischemia mitochondria, whereas the same concentration of metformin did not acutely inhibit complex I before the hypoxic period in cells or in mitochondria isolated from nonischemic hearts (Fig. 1, A and C). These results support that acute, high-dose metformin selectively downregulates complex I activity following the hypoxic or ischemic insult. This finding is in line with inhibition of the deactive form of complex I. The results also provide the foundation for the study of acute, high-dose metformin treatment in cardiac protection during early reperfusion.

Metformin Treatment at RO Protected Against Cardiac Cell Injury Following Simulated Ischemia. To assess the effect of metformin on cell death, H9c2 cells were exposed to 6-hour SI and 24-hour RO and treated with or without high-concentration metformin $(1 \mathrm{mM})$ at the onset of RO
(Fig. 2A). Strikingly, metformin, applied for only 30 minutes at RO, reduced cell death measured at 24-hour RO (Fig. 2A). The protection was similar to that observed when metformin was present during the entire 24-hour RO period (Fig. 2E). Metformin treatment decreased cell death following SI/RO evaluated using the CytoTox-Fluor assay (Fig. 1A). Cell death was also measured using the propidium iodide-Annexin $\mathrm{V}$ assay assessed using flow cytometry (Supplemental Fig. 1). Metformin treatment at the onset of RO following 6-hour SI decreased cellular ROS generation compared with cells exposed to untreated SI/RO assessed by using the fluorescent probe $\mathrm{H}_{2}$ DCFDA (Fig. 2D). Thus, the key timing of protection derived from acute administration of high concentration metformin was in the early reperfusion period. This result guided subsequent experiments in the intact heart.

AMPK was robustly (phosphorylated AMPK threonine-172) activated in cells exposed to 6-hour SI, as shown by the phosphorylation of AMPK and its downstream effector acetyl-CoA carboxylase. AMPK remained phosphorylated in H9c2 cells following RO (Fig. 2, B and C). Thus, acute, high-dose metformin treatment delivered at RO reduced cell death, although AMPK was already activated by the preceding period of hypoxia. These findings support a non-AMPK-mediated mechanism of action of acute, high-dose metformin therapy.

Metformin Treatment during Reperfusion Decreased Cardiac Injury. In isolated buffer-perfused mouse hearts, administration of high-concentration metformin at the onset of reperfusion for 5 minutes improved cardiac function, as shown by improved left ventricular developed pressure and decreased left ventricular end-diastolic pressure compared with untreated 

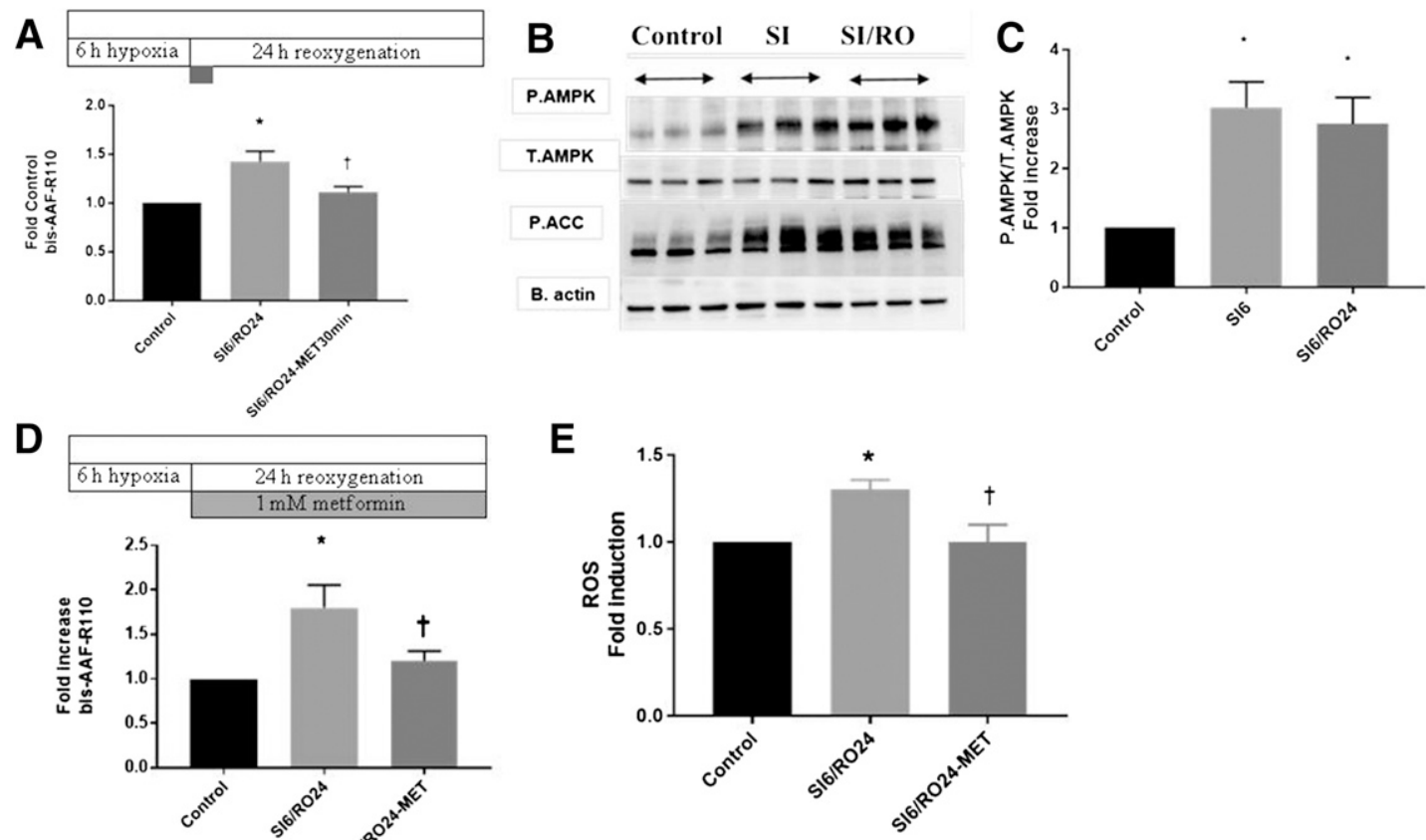

Fig. 2. Metformin treatment at the onset of RO decreased cell death in concert with selective inhibition of respiration through complex I. (A) Top metformin treatment of only 30 minutes at the onset of RO protected against cell death. Experimental protocol is shown. (A) Bottom: The result of the cytotoxicity assay is shown ( $n=3$ each group). (B) AMPK activation in three independent samples of H9c2 cells exposed to SI alone for 6-hour SI and 24-hour RO or normoxia alone (control). The AMPK downstream effector-phosphorylated acetyl-CoA carboxylase (P.ACC) is activated by SI or SI/RO, indicating functional AMPK activation. P.ACC is normalized to $\beta$ actin (B.actin). (C) Densitometry measurements from (B) of activated P-AMPK normalized to total AMPK. (D) Top: Scheme that represents the protocol of the experiments. (D) Bottom: Metformin protected H9c2 cells exposed to 6-hour SI and 24-hour RO against cell death ( $n=5$ in each group). (E) ROS production was measured in cells exposed to SI and RO (SI/RO) as in (D) ( $n=$ 3 each group). All data are expressed as mean \pm S.E.M.; ${ }^{*} P<0.05$ vs. control without SI; ${ }^{\dagger} P<0.05$ vs. untreated SI/RO.

hearts (Fig. 3, A and B). Metformin treatment also decreased the infarct size [mean \pm S.E.M., untreated ischemia reperfusion $(37 \% \pm 4 \%$ of left ventricle, $n=6)$ vs. $2 \mathrm{mM}$ metformin + ischemia reperfusion ( $15 \% \pm 3 \%$ of left ventricle, $n=6), P<0.05]$ measured by TTC staining (Fig. 3C). At the end of ischemia, in cytosol from mouse hearts, AMPK was robustly phosphorylated (Fig. 3, E and F), consistent with the findings in H9c2 cells following SI. Acute, high-dose metformin treatment delivered at the onset of reperfusion reduced cardiac injury, even though AMPK was already active due to the preceding period of ischemia. These results support that transient, high-dose metformin treatment at reperfusion decreases cardiac injury.

Metformin Treatment during Early Reperfusion Decreased MPTP Opening. CRC was used to assess the susceptibility to opening of the MPTP in isolated mitochondria (Paillard et al., 2009). The CRC in mitochondria from untreated ischemia-reperfused hearts was decreased compared with nonischemic hearts [mean \pm S.E.M., control (705 $\pm 20 \mathrm{nmol}$ calcium $/ \mathrm{mg}$ mitochondrial protein, $n=6$ ) vs. ischemia reperfusion ( $340 \pm 10, n=6), P<0.05$ ] (Fig. 3D). Metformin treatment at the onset of reperfusion significantly improved CRC compared with untreated hearts [mean \pm S.E.M., untreated ischemia reperfusion $(340 \pm 10 \mathrm{nmol}$ calcium $/ \mathrm{mg}$ mitochondrial protein, $n=6$ ) vs. $2 \mathrm{mM}$ metformin + ischemia reperfusion ( $467 \pm 48, n=6), P<0.05$ ] (Fig. 3D), indicating a decreased susceptibility to opening of the MPTP as a potential mechanism of cardiac protection resulting from the modulation of complex I activity at the onset of reperfusion.

The rate of oxidative phosphorylation was measured in mitochondria from buffer-perfused hearts with or without metformin treatment. Compared with nonischemic control, ischemia and reperfusion decreased the maximal rate of oxidative phosphorylation using both complex I and complex II substrates (Supplemental Table 1). Metformin treatment delivered at the onset of reperfusion did not improve oxidative phosphorylation compared with untreated hearts using complex I or complex II substrates (Supplemental Table 1). Metformin treatment did not alter the coupling of respiration nor state 4 ADP-limited respiration (Supplemental Table 1). Thus, the protection of metformin is not due to improved oxidative phosphorylation. The absence of improvement in respiration is in line with the previous observation that a substantial portion of damage to the ETC occurs during ischemia (Lesnefsky et al., 2004b), before high-dose metformin is administered. The absence of additional inhibition with metformin treatment is consistent with a reversibility of the acute, high-dose metformin effect over the 25 minutes of reperfusion between the cessation of metformin treatment and the study of mitochondria.

Metformin Given Only at the Onset of Reperfusion Decreased Infarct Size In Vivo. Wild-type mice (C57BL/6) underwent in vivo ischemia and reperfusion of the LAD territory with infarct size assessed at 24 hours after reperfusion. The risk area was similar in treated and untreated mice (Fig. 4A, left panel). Infarct size (Fig. 4A, right panel) was substantially decreased following a single treatment with metformin i.v. at the end of ischemia dosed to provide an approximately $2 \mathrm{mM}$ concentration in blood volume at the onset of reperfusion [mean \pm S.E.M.: vehicle $(0.20 \pm 0.02$ infarct size per total area at risk, $n=6)$ vs. metformin $(0.12 \pm 0.02 n=6), P<0.05]$. 

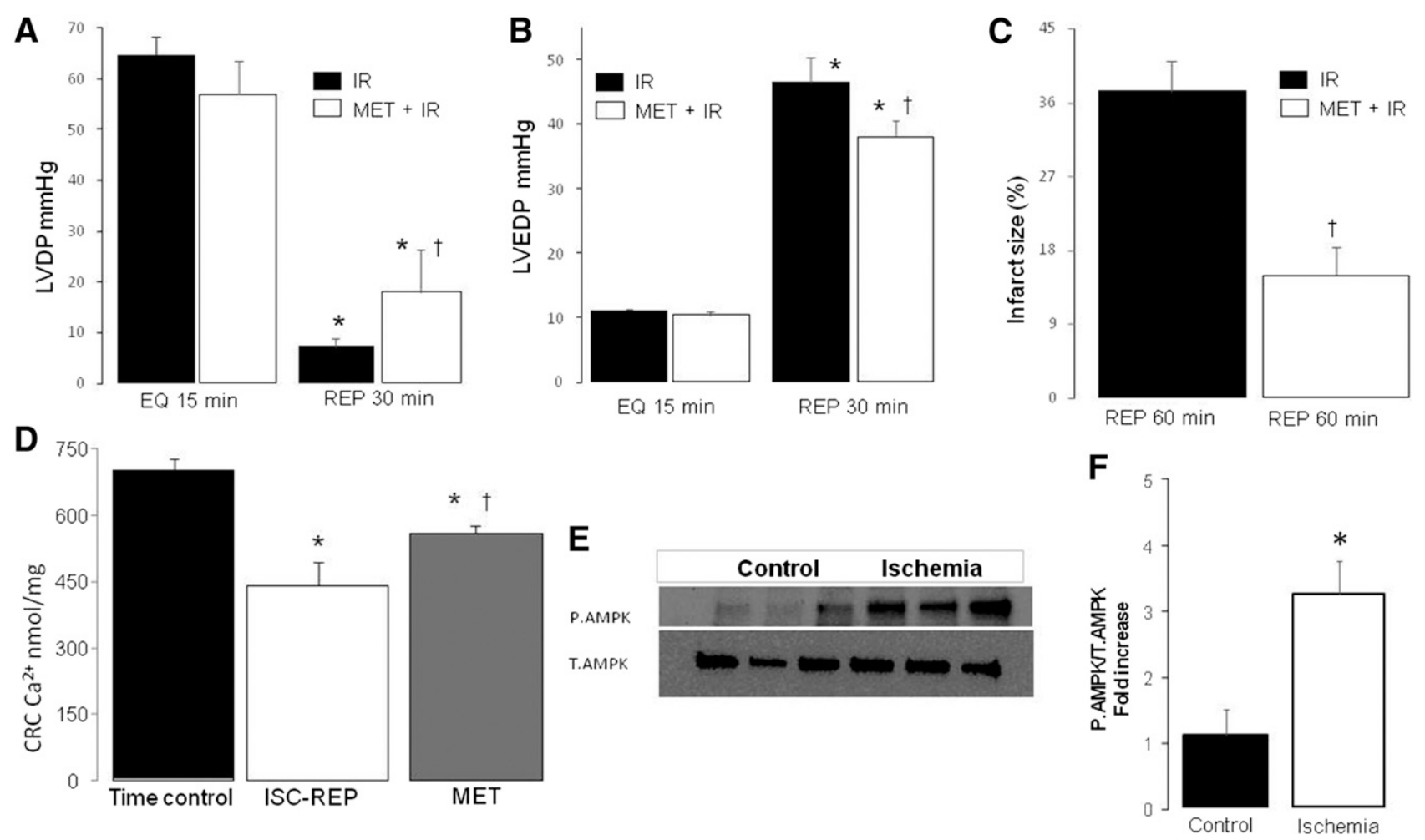

Fig. 3. Metformin treatment only during reperfusion decreased cardiac injury. (A and B) In isolated hearts: compared with untreated, metformin (MET) treatment improved the recovery of left ventricular developed pressure (LVDP, $\mathrm{mm} \mathrm{Hg}$ ) and decreased left ventricular end-diastolic pressure (LVEDP, $\mathrm{mm} \mathrm{Hg}$ ) during reperfusion (EQ, equilibration period before ischemia; REP, reperfusion). (C) Metformin treatment decreased myocardial infarct size compared with untreated hearts, indicating that acute, high-dose metformin treatment only at the onset of reperfusion markedly decreased cardiac injury. (D) Metformin treatment improved the CRC of mitochondria isolated following ischemia and reperfusion compared with untreated hearts, indicating that treatment decreased the susceptibility to opening of the MPTP during reperfusion. (E and F) Phosphorylation of AMPK (AMPK Thr172) was increased in cytosol isolated from isolated perfused mouse hearts at the end of stop flow ischemia compared with nonischemic time controls. All data are expressed as mean \pm S.E.M.; $* P<0.05$ vs. control; ${ }^{\dagger} P<0.05$ vs. untreated; $n=6$ in each group except for $n=3$ in (E and F).

Thus, metformin, given at the onset of reperfusion in a dose to cause reversible, partial inhibition of complex I, decreased cardiac injury in the adult heart following 24 hours of reperfusion in vivo.

We next asked whether high-dose metformin administered at the onset of reperfusion would reduce injury in the AMPK $\mathrm{KD}$ mouse. Wild-type littermate controls were again treated with metformin at the end of ischemia. Metformin again reduced infarct size in concomitant wild-type mice serving as a control (Fig. 4B) [mean \pm S.E.M.: vehicle $(0.22 \pm 0.02$ infarct size per total area at risk, $n=5$ vs. metformin $(0.12 \pm 0.01 n=4) ; P<$ 0.05]. Of note, high-dose acute metformin treatment at the onset of reperfusion reduced infarct size in the AMPK KD mice [mean \pm S.E.M.: vehicle $(0.34 \pm 0.02$, infarct size per total area at risk, $n=4)$ vs. metformin $(0.16 \pm 0.01, n=5) ; P<0.05$ ] (Fig. 4C). Note that infarct sizes, especially in the untreated AMPK KD group, tended to be greater than in the corresponding wild-type mice, consistent with greater susceptibility to injury in the AMPK KD model. Despite the increased susceptibility to myocardial necrosis, acute, high-dose metformin therapy delivered only at reperfusion reduced infarct size. This finding supports an AMPK-independent mechanism of protection with acute, high-dose early reperfusion treatment.

\section{Discussion}

We found that acute, high-dose treatment with metformin selectively decreases complex I activity in ischemia-damaged mitochondria and in H9c2 cells following hypoxia. Although chronic therapy with low-dose metformin decreases cardiac injury via activation of AMPK (Calvert et al., 2008; Varjabedian et al., 2018), acute, high-dose metformin treatment only at the onset of reperfusion decreases cardiac injury in mouse hearts and $\mathrm{H} 9 \mathrm{c} 2$ cells even though robust AMPK activation is already present. Furthermore, acute, high-dose metformin treatment reduced infarct size in vivo in the cardiac AMPK KD mouse. Thus, protection from acute, high-dose metformin at the onset of reperfusion occurs through partial inhibition of complex I. Metformin treatment decreases the cardiac injury in part via decreased MPTP opening during reperfusion. Attenuation of MPTP opening is linked with protective modulation of complex I.

Substantial damage to electron transport occurs during ischemia (Galkin and Moncada, 2007; Galkin et al., 2009; Chen et al., 2017). Ischemic damage to mitochondria occurs in part via the ETC with production of ROS that damage the ETC itself (Turrens, 2003; Lesnefsky et al., 2004a). The damaged ETC, in turn, augments cardiac injury during reperfusion (Lesnefsky et al., 2017). Although treatment during reperfusion cannot reverse ischemic damage, modulation of the ischemiadamaged ETC during reperfusion decreases myocyte injury (Stewart et al., 2009; Xu et al., 2014). Modulation of the ETC at complex I reduces injury by decreasing the production of ROS (Ambrosio and Flaherty, 1992; Kevin et al., 2003) and attenuates ETC effector mechanisms of MPTP opening, 
A

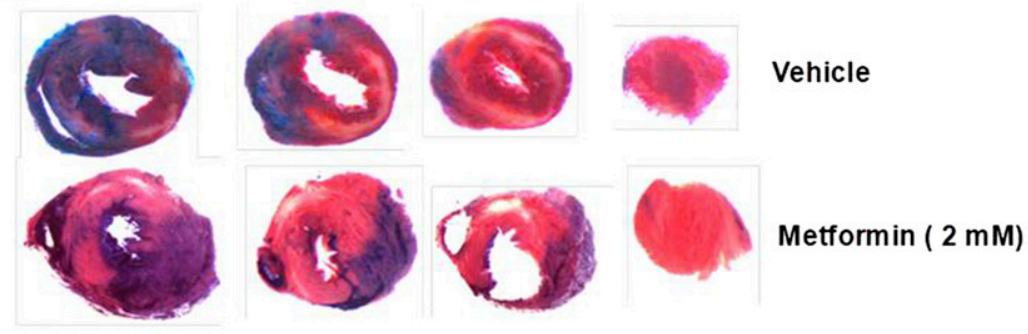

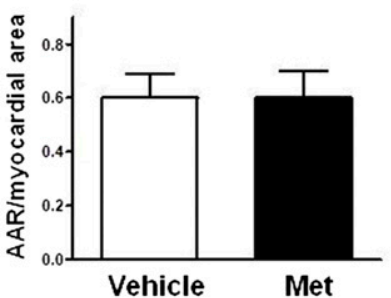

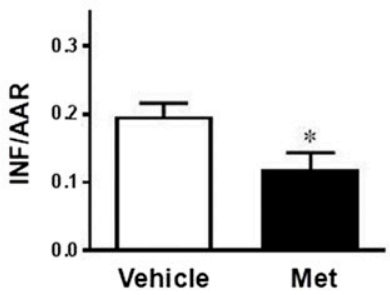

B
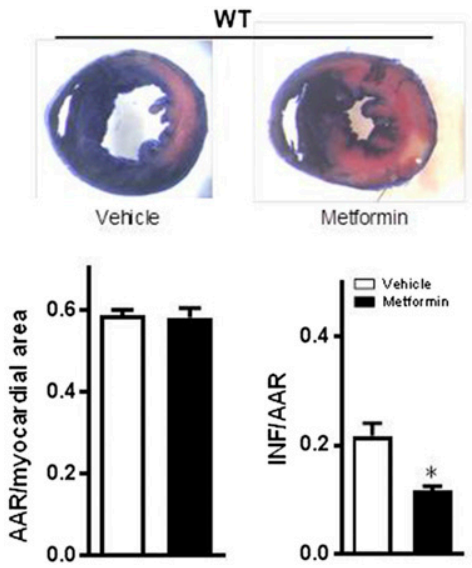

C
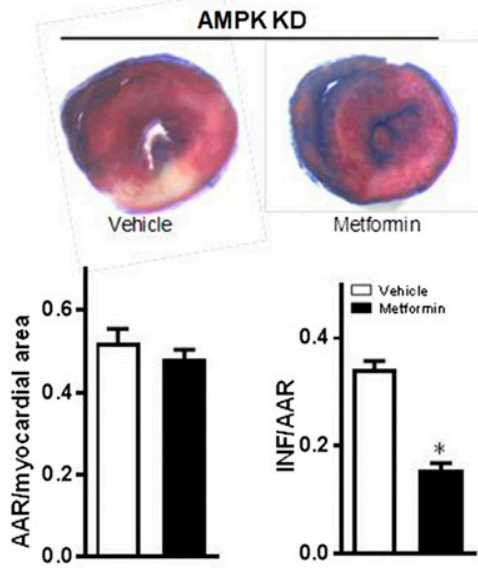

Fig. 4. Metformin given during reperfusion decreased cardiac injury in hearts in vivo. Mouse hearts underwent 45-minute LAD ligation and 24 hours of reperfusion. (A) Metformin treatment decreased the infarct size as a fraction of the ischemic zone area at risk (INF/AAR) in C57BL/6 mice following ischemia reperfusion $(n=6$ each group). The area at risk (AAR) ischemic zone was similar in each group (B and $\mathrm{C}$ ). Metformin treatment decreased infarct size in wild-type (B) and AMPK KD mice $(C)$ (wild type: vehicle $n=5$, metformin $n=4$; AMPK KD: vehicle $n=4$, metformin $n=5$ ). All data are expressed as mean \pm S.E.M.; $* P<0.05$ vs. untreated hearts. activation of mitochondrial proteases (Chen et al., 2011), and apoptosis (Chen et al., 2011). The optimal modulation of complex I appears to be a partial inhibition that does not lead to depolarization of mitochondrial membrane potential (Szczepanek et al., 2012). This biologic paradigm appears to be a balance that attenuates oxidative injury without substantially impairing ATP production.

Ischemia causes a functional defect within complex I that disrupts electron flow through the complex (Galkin and Moncada, 2007; Chen et al., 2008; Galkin et al., 2009). The key locus is near the coenzyme Q binding site (Galkin and Moncada, 2007; Chen et al., 2008; Galkin et al., 2009). Complex I exists in two different conformations, the active and the deactive conformations (Galkin and Moncada, 2007; Galkin et al., 2008, 2009; Babot and Galkin, 2013; Gorenkova et al., 2013), with the deactive form increased by ischemia (Galkin and Moncada, 2007; Galkin et al., 2009; Gorenkova et al., 2013). Deactive complex I is more sensitive to inhibition by biguanides (Bridges et al., 2014; Matsuzaki and Humphries, 2015). Two millimolar metformin acutely decreased complex I activity in ischemia-exposed mouse heart mitochondria, but not in mitochondria from nonischemic control hearts. Parallel experiments in H9c2 cells demonstrated acute inhibition of complex I respiration by metformin following hypoxia in contrast to normoxia. Our results thus suggest that the mechanism of complex I inhibition by metformin involves metformin-induced stabilization of the deactive form of complex I. These findings are particularly intriguing because they raise the potential for a reperfusion intervention that selectively targets the ischemia-damaged ETC that results in injury during early reperfusion.

Treatment with high-dose metformin at reperfusion decreased cardiac injury, in line with previous studies that reversible inhibition of complex I during reperfusion protects (Stewart et al., 2009; Szczepanek et al., 2012; Xu et al., 2014). Acute, high-dose metformin treatment to modulate complex I during early reperfusion decreased the production of ROS and decreased susceptibility of mitochondria to opening of the MPTP.

An increase in MPTP opening augments cardiac injury during reperfusion and is attenuated by transient blockade of electron transport at complex I (Baseler et al., 2013). Oxidative stress contributes to MPTP opening during reperfusion (Baseler et al., 2013). Metformin decreased ROS production measured during reoxygenation (Wang et al., 2017). ROS production during early reperfusion can occur by forward 
electron flow from complex I into complex III (Xu et al., 2014). ROS production also occurs at the onset of reperfusion via reverse electron flow from complex II into complex I in the presence of accumulated succinate (Chouchani et al., 2013, 2014; Pell et al., 2016) supported by the mitochondrial inner membrane potential. Reverse electron transport-mediated ROS generation sensitizes mitochondria to opening of the MPTP (Andrienko et al., 2017). Although the relative contribution of forward and reverse electron transport will vary based upon the presence of high concentration of succinate (onset of reperfusion) (Chouchani et al., 2014), extent of mitochondrial damage (ability to generate a membrane potential adequate to support reverse electron transport), issues that dynamically change as reperfusion continues, inhibition of complex I at the site distal in the complex attenuates ROS production from both forward and reverse electron transport. In addition, metformin treatment can decrease ROS generation in isolated hearts and $\mathrm{H} 9 \mathrm{c} 2$ cells following ischemia reperfusion by enhancing mitochondrial antioxidants, including manganese superoxide dismutase and catalase (Wang et al., 2017). Metformin may also affect other mitochondrial antioxidants, including thioredoxin reductase-2 (Stanley et al., 2011). Metformin may decrease MPTP opening through reduction of ROS generation via complex I inhibition or increased antioxidant capacity, protecting against reverse electron flow-mediated ROS generation at the onset of reperfusion and forward electron transport-mediated ROS from complex III as reperfusion continues.

Chronic, standard-dose metformin therapy activates AMPK by decreasing ATP content through partial inhibition of complex I (Bridges et al., 2014; Varjabedian et al., 2018). SI in H9c2 cells and stop flow ischemia in mouse hearts result in robust AMPK phosphorylation before RO/reperfusion. Thus, metformin is delivered to an already active AMPK system and nonetheless protects cardiomyocytes and intact hearts, although AMPK is already active before administration. The findings of the current study strongly support that the protective mechanism for metformin against early reperfusion is likely an AMPK-independent modulation of complex I.

In the diabetic patient receiving chronic metformin therapy, the metformin concentration usually is in the micromolar range (Babot and Galkin, 2013; Fontaine, 2014). In the present study, acute, physiologically significant modulation of complex I required 1 to $2 \mathrm{mM}$ metformin. Multiple previous studies found that various treatment paradigms, most involving chronic pretreatment before ischemia with standard (low)-dose metformin, can protect. Metformin protected both the diabetic and nondiabetic hearts during ischemia and reperfusion in mice (Calvert et al., 2008). It is of interest that treatment at the onset of reperfusion with lower-dose metformin than in the present study protected in an AMPKdependent manner because protection was not observed in the AMPK KD mouse (Calvert et al., 2008). In contrast, acute treatment at the onset of reperfusion in the current study with high-dose metformin protected the AMPK KD mouse. Reminiscent of the Calvert study, the study of Techiryan et al. (2018) in a swine model of infarction used a dose of metformin at reperfusion that achieved a peak plasma level of approximately $0.4 \mathrm{mM}$ at 15 minutes reperfusion. A reduction in infarct size was not observed (Techiryan et al., 2018). The dose and concentration used were likely below the 1 to $2 \mathrm{mM}$ needed to acutely inhibit complex I. This recent work further supports the role of acute modulation of complex I as a key mechanism of protection and the need for higher-dose metformin very early in reperfusion. There is clinical potential to use metformin as an acute complex I inhibitor based upon the paradigm of the current study. Administration of $2 \mathrm{mM}$ metformin intracoronary at the time of percutaneous intervention for STEMI for the initial 5 minutes of reperfusion would require a total dose of approximately $0.5 \mathrm{~g}$ of metformin, a clinically administered dose. This dose would equilibrate in the body following eventual release from the myocardium and based on previous pharmacokinetic data achieve the typical micromolar range concentration of chronic metformin therapy.

Acute, high-dose metformin can attenuate the ETC-driven injury that occurs early in reperfusion. This acute cardioprotection can be consolidated by the chronic use of standard-dose metformin to recruit AMPK-mediated cardioprotection during later recovery. Beneficial longer-term impacts of AMPKmediated signaling include the shift of substrate utilization toward glucose oxidation (Zaha and Young, 2012; Babot and Galkin, 2013), attenuation of endoplasmic reticulum stress (Chen et al., 2017), and the activation of protective mitophagy and mitochondrial biogenesis (Quan et al., 2018).

The current study builds upon previous work of cardioprotection achieved by the modulation of the ischemia-damaged ETC during reperfusion. Inhibition of ischemia-altered complex I with high-dose, acutely administered metformin, in relative preference to undamaged complex I, opens a new paradigm of targeted treatment of reperfusion injury. Metformin, a currently approved agent, could be repurposed for treatment. Lastly, the present study opens a novel therapeutic paradigm to treat reperfusion injury that will address the early phase of cardiac injury with the potential of continued therapy to activate additional cytoprotective mechanisms during later reperfusion. It is likely that successful clinical intervention against reperfusion injury will require complimentary therapy that approaches differing mechanisms of cardiac injury throughout the time course of myocardial recovery.

\section{Acknowledgments}

The critical review of the manuscript by Fadi Salloum (Pauley Heart Center, Virginia Commonwealth University) is appreciated.

\section{Authorship Contributions}

Participated in research design: Mohsin, Chen, Samidurai, Maceyka, Li, Lesnefsky.

Conducted experiments: Mohsin, Chen, Quan, Rousselle, Samidurai, Thompson, Hu.

Performed data analysis: Mohsin, Chen, Quan, Rousselle, Li, Samidurai, Lesnefsky.

Wrote or contributed to the writing of the manuscript: Mohsin, Chen, Maceyka, Rousselle, Quan, Samidurai, Li, Lesnefsky.

\section{References}

Ambrosio G and Flaherty JT (1992) Effects of the superoxide radical scavenger superoxide dismutase, and of the hydroxyl radical scavenger mannitol, on reperfusion injury in isolated rabbit hearts. Cardiovasc Drugs Ther 6:623-632.

Andrienko TN, Pasdois P, Pereira GC, Ovens MJ, and Halestrap AP (2017) The role of succinate and ROS in reperfusion injury - a critical appraisal. $J$ Mol Cell Cardiol 110:1-14.

Babot M and Galkin A (2013) Molecular mechanism and physiological role of activedeactive transition of mitochondrial complex I. Biochem Soc Trans 41:1325-1330. Baseler WA, Dabkowski ER, Jagannathan R, Thapa D, Nichols CE, Shepherd DL, Croston TL, Powell M, Razunguzwa TT, Lewis SE, et al. (2013) Reversal of mitochondrial proteomic loss in type 1 diabetic heart with overexpression of phospholipid hydroperoxide glutathione peroxidase. Am J Physiol Regul Integr Comp Physiol 304:R553-R565.

Bridges HR, Jones AJ, Pollak MN, and Hirst J (2014) Effects of metformin and other biguanides on oxidative phosphorylation in mitochondria. Biochem J 462:475-487. 
Calvert JW, Gundewar S, Jha S, Greer JJ, Bestermann WH, Tian R, and Lefer DJ (2008) Acute metformin therapy confers cardioprotection against myocardial infarction via AMPK-eNOS-mediated signaling. Diabetes 57:696-705

Chen Q, Moghaddas S, Hoppel CL, and Lesnefsky EJ (2008) Ischemic defects in the electron transport chain increase the production of reactive oxygen species from isolated rat heart mitochondria. Am J Physiol Cell Physiol 294:C460-C466.

Chen Q, Paillard M, Gomez L, Ross T, Hu Y, Xu A, and Lesnefsky EJ (2011) Activation of mitochondrial $\mu$-calpain increases AIF cleavage in cardiac mitochondria during ischemia-reperfusion. Biochem Biophys Res Commun 415:533-538.

Chen Q, Thompson J, Hu Y, Das A, and Lesnefsky EJ (2017) Metformin attenuates ER stress-induced mitochondrial dysfunction. Transl Res 190:40-50.

Chouchani ET, Methner C, Nadtochiy SM, Logan A, Pell VR, Ding S, James AM, Cochemé HM, Reinhold J, Lilley KS, et al. (2013) Cardioprotection by S-nitrosation of a cysteine switch on mitochondrial complex I. Nat Med 19:753-759.

Chouchani ET, Pell VR, Gaude E, Aksentijević D, Sundier SY, Robb EL, Logan A Nadtochiy SM, Ord ENJ, Smith AC, et al. (2014) Ischaemic accumulation of succinate controls reperfusion injury through mitochondrial ROS. Nature 515:431-435.

Das A, Xi L, and Kukreja RC (2005) Phosphodiesterase-5 inhibitor sildenafil preconditions adult cardiac myocytes against necrosis and apoptosis: essential role of nitric oxide signaling. $J$ Biol Chem 280:12944-12955.

Fontaine E (2014) Metformin and respiratory chain complex I: the last piece of the puzzle? Biochem J 463:e3-e5

Galkin A, Abramov AY, Frakich N, Duchen MR, and Moncada S (2009) Lack of oxygen deactivates mitochondrial complex I: implications for ischemic injury? $J$ Biol Chem 284:36055-36061.

Galkin A, Meyer B, Wittig I, Karas M, Schägger H, Vinogradov A, and Brandt U (2008) Identification of the mitochondrial ND3 subunit as a structural component involved in the active/deactive enzyme transition of respiratory complex I. J Biol Chem 283:20907-20913.

Galkin A and Moncada S (2007) S-nitrosation of mitochondrial complex I depends on its structural conformation. J Biol Chem 282:37448-37453.

Gomez L, Thibault H, Gharib A, Dumont JM, Vuagniaux G, Scalfaro P, Derumeaux G, and Ovize M (2007) Inhibition of mitochondrial permeability transition improves functional recovery and reduces mortality following acute myocardial infarction in mice. Am J Physiol Heart Circ Physiol 293:H1654-H1661.

Gorenkova N, Robinson E, Grieve DJ, and Galkin A (2013) Conformational change of mitochondrial complex I increases ROS sensitivity during ischemia. Antioxid Redox Signal 19:1459-1468.

Gustafsson AB and Gottlieb RA (2008) Heart mitochondria: gates of life and death. Cardiovasc Res 77:334-343.

Jochmans D, Leyssen P, and Neyts J (2012) A novel method for high-throughput screening to quantify antiviral activity against viruses that induce limited CPE. $J$ Virol Methods 183:176-179.

Kevin LG, Camara AK, Riess ML, Novalija E, and Stowe DF (2003) Ischemic preconditioning alters real-time measure of $\mathrm{O} 2$ radicals in intact hearts with ischemia and reperfusion. Am J Physiol Heart Circ Physiol 284:H566-H574.

Lesnefsky EJ, Chen Q, Moghaddas S, Hassan MO, Tandler B, and Hoppel CL (2004a) Blockade of electron transport during ischemia protects cardiac mitochondria. J Biol Chem 279:47961-47967.

Lesnefsky EJ, Chen Q, Slabe TJ, Stoll MS, Minkler PE, Hassan MO, Tandler B, and Hoppel CL (2004b) Ischemia, rather than reperfusion, inhibits respiration through cytochrome oxidase in the isolated, perfused rabbit heart: role of cardiolipin. Am J Physiol Heart Circ Physiol 287:H258-H267.

Lesnefsky EJ, Chen Q, Tandler B, and Hoppel CL (2017) Mitochondrial dysfunction and myocardial ischemia-reperfusion: implications for novel therapies. Annu Rev Pharmacol Toxicol 57:535-565.

Ma H, Wang J, Thomas DP, Tong C, Leng L, Wang W, Merk M, Zierow S, Bernhagen J, Ren J, et al. (2010) Impaired macrophage migration inhibitory factor-AMP-activated protein kinase activation and ischemic recovery in the senescent heart. Circulation 122:282-292.

Mamputu JC, Wiernsperger NF, and Renier G (2003) Antiatherogenic properties of metformin: the experimental evidence. Diabetes Metab 29:6S71-6S76.

Matsuzaki S and Humphries KM (2015) Selective inhibition of deactivated mitochondrial complex I by biguanides. Biochemistry 54:2011-2021.

Murphy E (2004) Primary and secondary signaling pathways in early preconditioning that converge on the mitochondria to produce cardioprotection. Circ Res 94:7-16.
Paillard M, Gomez L, Augeul L, Loufouat J, Lesnefsky EJ, and Ovize M (2009) Postconditioning inhibits mPTP opening independent of oxidative phosphorylation and membrane potential. J Mol Cell Cardiol 46:902-909.

Pell VR, Chouchani ET, Frezza C, Murphy MP, and Krieg T (2016) Succinate metabolism: a new therapeutic target for myocardial reperfusion injury. Cardiovasc Res 111:134-141.

Quan N, Sun W, Wang L, Chen X, Bogan JS, Zhou X, Cates C, Liu Q, Zheng Y, and Li J (2017) Sestrin2 prevents age-related intolerance to ischemia and reperfusion injury by modulating substrate metabolism. FASEB $J$ 31:4153-4167.

Quan N, Wang L, Chen X, Luckett C, Cates C, Rousselle T, Zheng Y, and Li J (2018) Sestrin2 prevents age-related intolerance to post myocardial infarction via AMPK/ PGC-1 $\alpha$ pathway. J Mol Cell Cardiol 115:170-178.

Russell RR III, Li J, Coven DL, Pypaert M, Zechner C, Palmeri M, Giordano FJ, Mu J, Birnbaum MJ, and Young LH (2004) AMP-activated protein kinase mediates ischemic glucose uptake and prevents postischemic cardiac dysfunction, apoptosis, and injury. $J$ Clin Invest 114:495-503.

Stanley BA, Sivakumaran V, Shi S, McDonald I, Lloyd D, Watson WH, Aon MA and Paolocci N (2011) Thioredoxin reductase-2 is essential for keeping low levels of $\mathrm{H}(2) \mathrm{O}(2)$ emission from isolated heart mitochondria. J Biol Chem 286:33669-33677.

Steel R and Torrie J (1960) Principles and Procedures of Statistics, McGraw-Hill, New York

Stewart S, Lesnefsky EJ, and Chen Q (2009) Reversible blockade of electron transport with amobarbital at the onset of reperfusion attenuates cardiac injury. Transl Res 153:224-231.

Strub GM, Paillard M, Liang J, Gomez L, Allegood JC, Hait NC, Maceyka M, Price MM, Chen Q, Simpson DC, et al. (2011) Sphingosine-1-phosphate produced by sphingosine kinase 2 in mitochondria interacts with prohibitin 2 to regulate complex IV assembly and respiration. FASEB J 25:600-612.

Szczepanek K, Chen Q, Derecka M, Salloum FN, Zhang Q, Szelag M, Cichy J, Kukreja RC, Dulak J, Lesnefsky EJ, et al. (2011) Mitochondrial-targeted signal transducer and activator of transcription 3 (STAT3) protects against ischemiainduced changes in the electron transport chain and the generation of reactive oxygen species. J Biol Chem 286:29610-29620.

Szczepanek K, Chen Q, Larner AC, and Lesnefsky EJ (2012) Cytoprotection by the modulation of mitochondrial electron transport chain: the emerging role of mitochondrial STAT3. Mitochondrion 12:180-189.

Techiryan G, Weil BR, Palka BA, and Canty JM Jr (2018) Effect of intracoronary metformin on myocardial infarct size in swine. Circ Res 123:986-995.

Turrens JF (2003) Mitochondrial formation of reactive oxygen species. J Physiol 552:335-344.

Varjabedian L, Bourji M, Pourafkari L, and Nader ND (2018) Cardioprotection by metformin: beneficial effects beyond glucose reduction. Am $J$ Cardiovasc Drugs 18:181-193.

Wang X, Yang L, Kang L, Li J, Yang L, Zhang J, Liu J, Zhu M, Zhang Q, Shen Y, et al. (2017) Metformin attenuates myocardial ischemia-reperfusion injury via up-regulation of antioxidant enzymes. PLoS One 12:e0182777.

Weiss JN, Korge P, Honda HM, and Ping P (2003) Role of the mitochondrial permeability transition in myocardial disease. Circ Res 93:292-301.

Xu A, Szczepanek K, Maceyka MW, Ross T, Bowler E, Hu Y, Kenny B, Mehfoud C, Desai PN, Baumgarten CM, et al. (2014) Transient complex I inhibition at the onset of reperfusion by extracellular acidification decreases cardiac injury. Am $J$ Physiol Cell Physiol 306:C1142-C1153.

Yang H, Sun W, Quan N, Wang L, Chu D, Cates C, Liu Q, Zheng Y, and Li J (2016) Cardioprotective actions of Notch1 against myocardial infarction via LKB1dependent AMPK signaling pathway. Biochem Pharmacol 108:47-57.

Yellon DM and Hausenloy DJ (2007) Myocardial reperfusion injury. $N$ Engl J Med 357:1121-1135.

Zaha VG and Young LH (2012) AMP-activated protein kinase regulation and biological actions in the heart. Circ Res 111:800-814.

Address correspondence to: Dr. Edward J. Lesnefsky, Cardiology Section, Medical Service 111(J), McGuire VA Medical Center, 1201 Broad Rock Boulevard, Richmond, VA 23249. E-mail: edward.lesnefsky@va.gov 